\title{
A Hybrid Contraction that Involves Jaggi Type
}

\author{
Erdal Karapınar 1,*D and Andreea Fulga 2,*(D) \\ 1 Department of Medical Research, China Medical University Hospital, China Medical University, \\ Taichung 40402, Taiwan \\ 2 Department of Mathematics and Computer Sciences, Universitatea Transilvania Brasov, \\ 500091 Brasov, Romania \\ * Correspondence: karapinar@mail.cmuh.org.tw or erdalkarapinar@yahoo.com (E.K.); afulga@unitbv.ro (A.F.)
}

Received: 24 April 2019; Accepted: 24 May 2019; Published: 27 May 2019

\begin{abstract}
In this manuscript, we aim to provide a new hybrid type contraction that is a combination of a Jaggi type contraction and interpolative type contraction in the framework of complete metric spaces. We investigate the existence and uniqueness of such a hybrid contraction in separate theorems. We consider a solution to certain fractional differential equations as an application of the given results. In addition, we provide an example to indicate the genuineness of the given results.
\end{abstract}

Keywords: hybrid contraction; Jaggi type contraction; fixed point; metric space; weight inequalities

MSC: 46T99; 47H10; 54H25

\section{Introduction and Preliminaries}

Fixed point theory is based on the solution of a simple (fixed point) equation, $f(x)=x$, for a self-mapping $f$ on a non-empty set $X$. Inherently, a fixed point equation $f(x)=x$ can be reformulated as $F(x)=f(x)-x=0$, where $F$ is also a self-mapping on $X$. The fixed point problem first appeared in the solution of a differential equation with an initial value. In 1837, Liouville [1] solved such differential equations by employing the method of successive approximations which implicitly brings a solution for thefixed point equation. In 1890, Picard [2] developed the aforementioned method systematically and simplified the solution of the corresponding differential equation. Later, Banach [3] was able to derive the abstraction of the method of successive approximation so that he proved a celebrated fixed point theorem in the framework of a complete metric space. After that, this outstanding result from Banach was characterized by Caccioppoli [4] in the context of complete metric spaces: Every contraction in a complete metric space possesses a unique fixed point. This result has been called several names, such as Picard-Banach fixed point theorem, Banach-Caccioppoli fixed point theorem, Banach's contraction (mapping principle), and Banach's fixed point theorem. Throughout this paper, we refer to it as "Banach-Caccioppoli fixed point theorem". For some recent and enhanced results, see e.g., [5,6].

Banach-Caccioppoli fixed point theorem initiated metric fixed point theory which has attracted the attention of researchers in nonlinear functional analysis. In the last decades, a number of fixed point results have been published to extend and generalize Banach-Caccioppoli fixed point theorem. Among them, we focus on two fixed point theorems that belong to Jaggi [7] and Karapinar [8]. Jaggi [7] proved a fixed point theorem in which the rational expression was considered for the first time. In addition, Karapinar [8] introduced the notion of an interpolative type contraction to enrich metric fixed point theory by linking the interpolation theory with it (see also [9-13]). Very recently, inspired by the result in [8], Mitrović et al. [14] introduced and investigated a hybrid contraction that combines a Reich type contraction and interpolative type contractions. 
In this paper, we have two main motivations. First, we shall introduce and investigate a new hybrid contraction that combines Jaggi type contractions and interpolative type contractions. Regarding the appearance of fixed point theory, it is natural to consider the solution of differential equations using fixed point theory as an application. On the other hand, recently, fractional calculus and fractional differential equations have been studied intensely. By taking this observation into account, as a second aim, we propose a solution to certain fractional differential equations by employing our main results. We also consider an example to show the validity of our main results.

In what follows, we review some basic notions. We say that $\psi:[0, \infty) \rightarrow[0, \infty)$ is a (c)-comparison function (see $[15,16])$ if it is nondecreasing and satisfies

(\$) there are $l_{0} \in \mathbb{N}$ and $k \in(0,1)$ and a convergent series $\sum_{l=1}^{\infty} z_{l}$ such that $z_{l} \geq 0$ and

$$
\psi^{l+1}(t) \leq k \psi^{l}(t)+z_{l}
$$

for $l \geq l_{0}$ and $t \geq 0$.

The set of all function (c)-comparison function will be denoted by $\Psi$. Every $\psi \in \Psi$ verifies the following [16]:

(a) $\quad \psi(z)<z$, for any $z \in \mathbb{R}^{+}$;

(b) $\psi$ is continuous at 0 ;

(c) the series $\sum_{l=1}^{\infty} \psi^{l}(z)$ is convergent for $z \geq 0$.

\section{Main Results}

In our next consideration, we suppose that $(\mathcal{M}, d)$ denotes a complete metric space.

Definition 1. A self-mapping $T$ on $(\mathcal{M}, d)$ is called a Jaggi type hybrid contraction if there is $\psi \in \Psi$ so that

$$
d(T x, T y) \leq \psi\left(g_{T}^{s}(x, y)\right)
$$

for all distinct $x, y \in \mathcal{M}$ where $s \geq 0$ and $\sigma_{i} \geq 0, i=1,2$, such that $\sigma_{1}+\sigma_{2}=1$ and

$$
g_{T}^{s}(x, y)= \begin{cases}{\left[\sigma_{1}\left(\frac{d(x, T x) \cdot d(y, T y)}{d(x, y)}\right)^{s}+\sigma_{2}(d(x, y))^{s}\right]^{1 / s},} & \text { for } s>0, \quad x, y \in \mathcal{M}, x \neq y \\ (d(x, T x))^{\sigma_{1}}(d(y, T y))^{\sigma_{2}}, \quad \text { for } s=0, \quad x, y \in \mathcal{M} \backslash \mathcal{F}_{T}(\mathcal{M})\end{cases}
$$

where $\mathcal{F}_{T}(\mathcal{M})=\{z \in \mathcal{M}: T z=z\}$.

Theorem 1. A continuous self-mapping $T$ on $(\mathcal{M}, d)$ possesses a fixed point $x$ provided that $T$ is a Jaggi type hybrid contraction. Moreover, for any $\chi_{0} \in \mathcal{M}$, the sequence $\left\{T^{n} \chi_{0}\right\}$ converges to $\chi$.

Proof. For an arbitrary $x$ in $\mathcal{M}$, renamed as $x=x_{0}$, we set-up an iterative sequence $\left\{x_{n}\right\}$ by starting this initial point $x_{0}$ defined as follows:

$$
x_{1}=T x_{0}, x_{2}=T x_{1}=T^{2} x_{0} \ldots, x_{n}=T x_{n-1}=T^{n-1} x_{0} .
$$

We presume that

$$
d\left(x_{n}, x_{n+1}\right)>0 \text { for all } n \in \mathbb{N}_{0} .
$$

On the contrary, if the inequality above does not hold, that is, if there exists $k_{0} \in \mathbb{N}_{0}$ such that $d\left(x_{k_{0}}, \chi_{k_{0}+1}\right)=0$, then we get $x_{k_{0}}=x_{k_{0}+1}=T \chi_{k_{0}}$. Since $x_{k_{0}}$ forms a fixed point, it terminates the proof.

We shall prove the claim of the theorem by examining two distinct cases: $s=0$ and $s>0$. 
(i) In the case where $s>0$, by letting $g_{T}^{s}(x, y)$ for $x=x_{n-1}$ and $y=T x_{n-1}$, the expression (2) becomes

$$
d\left(T x_{n-1}, T\left(T x_{n-1}\right)\right) \leq \psi\left(g_{T}^{S}\left(x_{n}, x_{n+1}\right)\right)
$$

where

$$
\begin{aligned}
g_{T}^{s}\left(x_{n-1}, T \chi_{n-1}\right) & =\left[\sigma_{1}\left(\frac{d\left(x_{n-1}, T x_{n-1}\right) \cdot d\left(T x_{n-1}, T^{2} x_{n-1}\right)}{d\left(x_{n-1}, T x_{n}-1\right)}\right)^{s}+\sigma_{2}\left(d\left(x_{n-1}, T x_{n-1}\right)\right)^{s}\right]^{1 / s}, \\
& =\left[\sigma_{1}\left(\frac{d\left(x_{n-1}, x_{n}\right) \cdot d\left(x_{n}, x_{n}\right)}{d\left(x_{n-1}, x_{n}\right)}\right)^{s}+\sigma_{2}\left(d\left(x_{n-1}, x_{n}\right)\right)^{s}\right]^{1 / s}, \\
& =\left[\sigma_{1}\left(d\left(x_{n}, x_{n+1}\right)\right)^{s}+\sigma_{2}\left(d\left(x_{n-1}, x_{n}\right)\right)^{s}\right]^{1 / s} .
\end{aligned}
$$

Therefore, we have

$$
d\left(T x_{n-1}, T\left(T x_{n-1}\right)\right) \leq \psi\left(g_{T}^{s}\left(x_{n}, x_{n+1}\right)\right)=\psi\left(\left[\sigma_{1}\left(d\left(x_{n}, x_{n+1}\right)\right)^{s}+\sigma_{2}\left(d\left(x_{n-1}, x_{n}\right)\right)^{s}\right]^{1 / s}\right) .
$$

Suppose that $d\left(x_{n}, x_{n+1}\right) \geq d\left(x_{n-1}, x_{n}\right)$, then, from the inequality (5), we find

$$
\begin{aligned}
d\left(x_{n}, x_{n+1}\right) & \leq \psi\left(\left[\sigma_{1}\left(d\left(x_{n}, x_{n+1}\right)\right)^{s}+\sigma_{2}\left(d\left(x_{n-1}, x_{n}\right)\right)^{s}\right]^{1 / s}\right) \\
& \leq \psi\left(\left[\sigma_{1}\left(d\left(x_{n}, x_{n+1}\right)\right)^{s}+\sigma_{2}\left(d\left(x_{n}, x_{n+1}\right)\right)^{s}\right]^{1 / s}\right) \\
& \leq \psi\left(\left[\left(\sigma_{1}+\sigma_{2}\right)\left(d\left(x_{n}, x_{n+1}\right)\right)^{s}\right]^{1 / s}\right) \\
& =\psi\left(\left[\left(d\left(x_{n}, x_{n+1}\right)\right)^{s}\right]^{1 / s}\right) \\
& =\psi\left(d\left(x_{n}, x_{n+1}\right)\right) \\
& <d\left(x_{n}, x_{n+1}\right),
\end{aligned}
$$

a contradiction. Hence, we obtain that

$$
d\left(x_{n}, x_{n+1}\right) \leq d\left(x_{n-1}, x_{n}\right) .
$$

By revisiting inequality (5), we find that

$$
\left.d\left(x_{n}, x_{n+1}\right)\right) \leq \psi\left(d\left(x_{n-1}, x_{n}\right)\right)<d\left(x_{n-1}, x_{n}\right) .
$$

Continuing in this way, we deduce (inductively) that

$$
d\left(x_{n}, x_{n+1}\right) \leq \psi^{n}\left(d\left(x_{0}, x_{1}\right)\right),
$$

for any $n \in \mathbb{N}_{0}$. Now, let $p \in \mathbb{N}_{0}$. By using the triangle inequality and (7), we have

$$
\begin{aligned}
d\left(x_{n}, x_{n+p}\right) & \leq d\left(x_{n}, x_{n+1}\right)+d\left(x_{n+1}, x_{n+p}\right) \\
& \leq d\left(x_{n}, x_{n+1}\right)+d\left(x_{n+1}, x_{n+2}\right)+\ldots+d\left(x_{n+l-1}, x_{n+l}\right) \\
& \leq \sum_{k=n}^{n+p-1} \psi^{k}\left(d\left(x_{0}, x_{1}\right)\right) \leq \sum_{k=n}^{\infty} \psi^{k}\left(d\left(x_{0}, x_{1}\right)\right) .
\end{aligned}
$$

Letting $n \rightarrow \infty$ in (8) and in accordance with the properties of (c)-comparison functions, we get that $\left.\lim _{n \rightarrow \infty} d\left(\chi_{n}, \chi_{n+p}\right)\right)=0$. Thereby, $\left\{\chi_{n}\right\}$ is a Cauchy sequence in a complete metric space $(\mathcal{M}, d)$, so that there exists $x \in X$ such that

$$
\lim _{n \rightarrow \infty} d\left(x_{n}, x\right)=0 .
$$

Since $T$ is a continuous map, it follows that

$$
d(\chi, T \chi)=\lim _{n \rightarrow \infty} d\left(x_{n}, T x_{n}\right)=\lim _{n \rightarrow \infty} d\left(x_{n}, x_{n+1}\right)=0,
$$

which shows that $x$ is a fixed point of $T$. 
(ii) In the case where $s=0$, using (2) for $x=x_{n-1}$ and $y=T x_{n-1}$, we have

$$
\begin{aligned}
d\left(x_{n}, x_{n+1}\right) & =d\left(T x_{n-1}, T\left(T x_{n-1}\right)\right) \leq \psi\left(g_{T}^{s}\left(x_{n-1}, T x_{n-1}\right)\right) \\
& \left.=\psi\left(d\left(x_{n-1}, T x_{n-1}\right)\right)^{\sigma_{1}}\left(d\left(x_{n}, T x_{n-1}\right)\right)^{\sigma_{2}}\right) \\
& <\left(d\left(x_{n-1}, x_{n}\right)\right)^{\sigma_{1}}\left(d\left(x_{n}, x_{n+1}\right)\right)^{\sigma_{2}} .
\end{aligned}
$$

Using a simple calculation, the above inequality turns into

$$
\left.d\left(x_{n}, x_{n+1}\right)^{1-\sigma_{2}}<d\left(x_{n-1}, x_{n}\right)\right)^{\sigma_{1}},
$$

and since $\sigma_{1}+\sigma_{2}=1$, we get that $d\left(\chi_{n}, x_{n+1}\right)<d\left(x_{n-1}, x_{n}\right)$ for all $n \in \mathbb{N}_{0}$. Returning to (10), we find

$$
d\left(x_{n}, x_{n+1}\right) \leq \psi\left(d\left(x_{n-1}, x_{n}\right)\right)
$$

and by inductive reasoning

$$
d\left(x_{n}, x_{n+1}\right) \leq \psi^{n}\left(d\left(x_{0}, x_{1}\right)\right) .
$$

By using the same tools as in the case of $s>0$, we can easily find that $\left\{x_{n}\right\}$ forms a Cauchy sequence in a complete metric space. Hence, there exists $x \in \mathcal{M}$ such that $\lim _{n \rightarrow \infty} d\left(x_{n}, x\right)=0$ and from the continuity of $T$, we get that this point is a fixed point of mapping $T$.

In what follows, we intend to prove the existence of a fixed point of a map $T$ under the presumption of continuity only of some iterate of $T$.

Theorem 2. Let $(\mathcal{M}, d)$ be a complete metric space and $T: \mathcal{M} \rightarrow \mathcal{M}$ be a Jaggi type hybrid contraction. In the case where for some integer $p>1, T^{p}$ is continuous then $T$ has a unique fixed point.

Proof. From the proof of Theorem 1, we already know that the sequence $\left\{x_{n}\right\}$, with $x_{n}=T x_{n-1}$ for all $n \in \mathbb{N}_{0}$ is convergent to some point $x \in \mathcal{M}$, that is $d\left(x_{n}, x\right)=0$. Let $\left\{x_{n}(l)\right\}$ be a subsequence of $\left\{x_{n}\right\}$, where $n(l)=l \cdot p$ for all $l \in \mathbb{N}_{0}$ and $p>1$ fixed. Thus, considering that $T^{0}$ is the identity map on $X$, we have $x_{n(l)}=T^{p} x_{n(l)-p}$. Then, due to continuity of $T^{p}$,

$$
d\left(x, T^{p} x\right)=\lim _{l \rightarrow \infty} d\left(x, T^{p} x_{n(l)-p}\right)=\lim _{l \rightarrow \infty} d\left(x_{n(l)}, x\right)=d(x, x)=0,
$$

which means that $\chi$ is a fixed point of $T^{p}$.

Let us presume that $T \chi \neq \chi$. We remark that, in this situation, $T^{p-k-1} \chi \neq T^{p-k} \chi$ for any $k=0,1, \ldots, p-1$. Again we must consider two cases.

(i) In the case where $s>0$, by replacing $x$ by $T^{p-k-1} \chi$ and $y$ by $T^{p-k} \chi$, we have

$$
\begin{aligned}
g_{T}^{s}\left(T^{p-k-1} \chi, T^{p-k} \chi\right) & =\left[\sigma_{1}\left(\frac{d\left(T^{p-k-1} \chi, T\left(T^{p-k-1} \chi\right)\right) \cdot d\left(T^{p-k} \chi T\left(T^{p-k} \chi\right)\right)}{d\left(T^{p-k-1} \chi T^{p-k} \chi\right.}\right)^{s}+\sigma_{2}\left(d\left(T^{p-k-1} \chi, T^{p-k} \chi\right)\right)^{s}\right. \\
& =\left[\sigma_{1}\left(d\left(T^{p-k} \chi, T^{p-k+1} \chi\right)\right)^{s}+\sigma_{2}\left(d\left(T^{p-k-1} \chi, T^{p-k} \chi\right)\right)^{s}\right]^{1 / s},
\end{aligned}
$$

and since $T$ is a Jaggi type hybrid contraction,

$$
\begin{aligned}
d\left(T^{p-k} \chi, T^{p-k+1} \chi\right) & =d\left(T\left(T^{p-k-1} \chi\right), T\left(T^{p-k} \chi\right)\right) \leq \psi\left(g_{T}^{s}\left(T^{p-k-1} \chi, T^{p-k} \chi\right)\right) \\
& =\psi\left(\left[\sigma_{1}\left(d\left(T^{p-k} \chi, T^{p-k+1} \chi\right)\right)^{s}+\sigma_{2}\left(d\left(T^{p-k-1} \chi, T^{p-k} \chi\right)\right)^{s}\right]^{1 / s}\right) \\
& <\left[\sigma_{1}\left(d\left(T^{p-k} \chi, T^{p-k+1} \chi\right)\right)^{s}+\sigma_{2}\left(d\left(T^{p-k-1} \chi, T^{p-k} \chi\right)\right)^{s}\right]^{1 / s},
\end{aligned}
$$


which yields that

$$
d\left(T^{p-k} \chi, T^{p-k+1}\right)^{s}\left(1-\sigma_{1}\right)<\sigma_{2}\left(d\left(T^{p-k-1} \chi, T^{p-k} \chi\right)\right)^{s} .
$$

However, $\sigma_{1}+\sigma_{2}=1$, so that for every $k=0,1, \ldots, p-1$

$$
d\left(T^{p-k} \chi, T^{p-k+1} \chi\right)<d\left(T^{p-k-1} \chi, T^{p-k} \chi\right) .
$$

Of course, the inequality (27) implies that for every $l=k, k+1, \ldots, p-1$,

$$
d\left(T^{p-k} \chi, T^{p-k+1} \chi\right)<d\left(T^{p-k-l-1} \chi, T^{p-k-l} \chi\right) .
$$

Taking in particular $k=0$ and $l=p-1$ in the above inequality, we get

$$
d\left(\chi, T^{p} \chi\right)=d\left(T^{p} \chi, T^{p+1} \chi\right)<d(\chi, T \chi),
$$

which is a contradiction. Consequently, $T \chi=\chi$.

(ii) In the case where $s=0$,

$$
\begin{aligned}
g_{T}^{s}\left(T^{p-k-1} \chi, T^{p-k} \chi\right) & =\left[d\left(T^{p-k-1} \chi, T\left(T^{p-k-1} \chi\right)\right)\right]^{\sigma_{1}}\left[d\left(T^{p-k} \chi, T\left(T^{p-k} \chi\right)\right)^{\sigma_{2}}\right] \\
& =\left[d\left(T^{p-k-1} \chi, T^{p-k} \chi\right)\right]^{\sigma_{1}}\left[d\left(T^{p-k} \chi, T^{p-k+1} \chi\right)\right]^{\sigma_{2}}
\end{aligned}
$$

and inequality (2) becomes

$$
\begin{aligned}
d\left(T^{p-k} \chi, T^{p-k+1} \chi\right) & =d\left(T\left(T^{p-k-1} \chi\right), T\left(T^{p-k} \chi\right)\right) \leq \psi\left(g_{T}^{s}\left(T^{p-k-1} \chi, T^{p-k} \chi\right)\right) \\
& =\psi\left(\left[d\left(T^{p-k-1} \chi, T^{p-k} \chi\right)\right]^{\sigma_{1}}\left[d\left(T^{p-k} \chi, T^{p-k+1} \chi\right)\right]^{\sigma_{2}}\right) \\
& <\left[d\left(T^{p-k-1} \chi, T^{p-k} \chi\right)\right]^{\sigma_{1}}\left[d\left(T^{p-k} \chi, T^{p-k+1} \chi\right)\right]^{\sigma_{2}} .
\end{aligned}
$$

By simple calculation, we get

$$
d\left(T^{p-k} \chi, T^{p-k+1} \chi\right)<d\left(T^{p-k-1} \chi, T^{p-k} \chi\right),
$$

and using the same tools as in the case (ii), we obtain that $T \chi=x$.

Example 1. Let $\mathcal{M}=[0,1]$, the distance

$$
d(z, t)=\left\{\begin{aligned}
\max \{z, t\}, & \text { if } z \neq t \\
0, & \text { if } z=t
\end{aligned}\right.
$$

and a mapping $T: \mathcal{M} \rightarrow \mathcal{M}$ be defined as

$$
T(z)= \begin{cases}0, & \text { for } z \in\left[0, \frac{1}{4}\right) \\ \frac{1}{8}, & \text { for } z \in\left[\frac{1}{4}, \frac{1}{2}\right] \\ \frac{1}{4}, & \text { for } z \in\left(\frac{1}{2}, 1\right] .\end{cases}
$$

The mapping $T$ is not continuous in $z=\frac{1}{4}$, neither in $z=\frac{1}{2}$, so Theorem 1 cannot be applied. It is easy to see that $T^{2}(z)=\left\{\begin{array}{ll}0, & \text { for } z \in\left[0, \frac{1}{2}\right] \\ \frac{1}{8}, & \text { for } z \in\left(\frac{1}{2}, 1\right]\end{array}\right.$ is not continuous in $z=\frac{1}{2}$, instead $T^{3}(z)=0$ is continuous on M. We claim that $T$ satisfies all the assumptions of Theorem 2. Letting $\sigma_{1}=\frac{1}{4}, \sigma_{2}=\frac{3}{4}$ and $s=2$, we have 


$$
g_{T}^{2}(z, t)=\left[\frac{1}{4}\left(\frac{d(z, T z) \cdot d(t, T t)}{d(z, t)}\right)^{2}+\frac{3}{4}\left(d(z, t)^{2}\right]^{1 / 2},\right.
$$

and for $\psi(t)=\frac{3}{4} t,(2)$ becomes

$$
d(T z, T t) \leq \psi\left(g_{T}^{2}(z, t)\right)=\frac{3}{4} \cdot\left[\frac{1}{4}\left(\frac{d(z, T z) \cdot d(t, T t)}{d(z, t)}\right)^{2}+\frac{3}{4}\left(d(z, t)^{2}\right]^{1 / 2} .\right.
$$

Let us denote $A=\left[0, \frac{1}{4}\right), B=\left[\frac{1}{4}, \frac{1}{2}\right)$ and $C=\left[\frac{1}{2}, 1\right]$. Of course, if $(z, t) \in\{A \times A, B \times B, C \times C\}$, Theorem 2 is satisfied directly. Therefore, we should examine the cases:

1. If $z \in A, t \in B$, then (17) yields

$$
\begin{aligned}
d(T z, T t) & =\frac{1}{8}<\frac{3}{4}\left[\frac{1}{4} z^{2}+\frac{3}{4} t^{2}\right]^{1 / 2}=\frac{3}{4}\left[\frac{1}{4}\left(\frac{\max \{z, 0\} \cdot \max \{t, 1 / 8\}}{\max \{z, t\}}\right)^{2}+\frac{3}{4}(d \max \{z, t\})^{2}\right]^{1 / 2} \\
& =\psi\left(g_{T}^{2}(z, t)\right)
\end{aligned}
$$

2. If $z \in A, t \in C$, then (17) yields

$$
\begin{aligned}
d(T z, T t) & =\frac{1}{4}<\frac{3}{4}\left[\frac{1}{4} z^{2}+\frac{3}{4} t^{2}\right]^{1 / 2}=\frac{3}{4}\left[\frac{1}{4}\left(\frac{\max \{z, 0\} \cdot \max \{t, 1 / 4\}}{\max \{z, t\}}\right)^{2}+\frac{3}{4}(d \max \{z, t\})^{2}\right]^{1 / 2} \\
& =\psi\left(g_{T}^{2}(z, t)\right)
\end{aligned}
$$

3. If $z \in B, t \in C$, then (17) yields

$$
\begin{aligned}
d(T z, T t) & =\frac{1}{8}<\frac{3}{4}\left[\frac{1}{4} z^{2}+\frac{3}{4} t^{2}\right]^{1 / 2}=\frac{3}{4}\left[\frac{1}{4}\left(\frac{\max \{z, 1 / 8\} \cdot \max \{t, 1 / 4\}}{\max \{z, t\}}\right)^{2}+\frac{3}{4}(d \max \{z, t\})^{2}\right]^{1 / 2} \\
& =\psi\left(g_{T}^{2}(z, t)\right) .
\end{aligned}
$$

Thus, since all the presumptions of Theorem 2 are satisfied, $T$ has a fixed point. Indeed, $z=0$ is the fixed point of $T$.

At the end of this section, we list some immediate consequences of the main theorem. Indeed, letting $\psi(z)=\lambda z, z \geq 0$ in Theorem 1 , for $p>0$ we have:

Corollary 1. A continuous self-mapping $T$ on $(\mathcal{M}, d)$ has a fixed point $x^{*}$ if for any $x, y \in \mathcal{M}, x \neq y$

$$
d(T x, T y) \leq \lambda\left[\sigma_{1}\left(\frac{d(x, T \chi) d(y, T y)}{d(x, y)}\right)^{s}+\sigma_{2}(d(x, y))^{s}\right]^{1 / s},
$$

where $\sigma_{1}, \sigma_{2} \geq 0$ with $\sigma_{1}+\sigma_{2}=1, s>0$ and $\lambda \in(0,1)$.

Corollary 2. A continuous self-mapping $T$ on $(\mathcal{M}, d)$ has a fixed point if for any $x, y \in \mathcal{M}, x \neq y$

$$
d(T x, T y) \leq \frac{\lambda}{\sqrt{2}}\left[\left(\frac{d(x, T \chi) d(y, T y)}{d(x, y)}\right)^{2}+(d(x, y))^{2}\right]^{1 / 2},
$$

where $\lambda \in(0,1)$.

Proof. Put in Corollary $1 \sigma_{1}=\sigma_{2}=\frac{1}{2}$ and $s=2$. 
Corollary 3. A self-mapping $T$ on $(\mathcal{M}, d)$ has a fixed point $x^{*}$ if for $x, y \in \mathcal{M} \backslash \mathcal{F}_{T}(\mathcal{M})$,

$$
d(T x, T y) \leq \lambda[d(x, T x)]^{\alpha}[d(y, T y)]^{1-\alpha},
$$

where $\alpha, \lambda \in(0,1)$.

Proof. It follows from Theorem 1 , letting $\psi(z)=\lambda z$ for any $z \geq 0, p=0$, respectively, $\sigma_{1}=\alpha, \sigma_{2}=$ $1-\alpha$.

Corollary 4. A self-mapping $T$ on $(\mathcal{M}, d)$ has a fixed point if it satisfies the inequality

$$
d(T x, T y) \leq \lambda \sqrt{d(x, T x) d(y, T y)}
$$

for $x, y \in \mathcal{M} \backslash \mathcal{F}_{T}(\mathcal{M})$, where $\lambda \in(0,1)$.

Proof. Put in Corollary $1 \alpha=1 / 2$.

If in Corollary 1 , we take $p=1, \alpha=\lambda \sigma_{1}, \beta=\lambda \sigma_{2}$, where $\lambda \in(0,1)$, we obtain the following:

Corollary 5. Let the space $(\mathcal{M}, d)$ and $T: \mathcal{M} \rightarrow \mathcal{M}$ be a continuous mapping such that for $x, y \in \mathcal{M}, x \neq y$,

$$
d(T x, T y) \leq \alpha \frac{d(x, T x) d(y, T y)}{d(x, y)}+\beta d(x, y),
$$

where $\alpha, \beta \in(0,1)$ with $\alpha+\beta<1$. Therefore, $T$ possesses a fixed point.

\section{Application}

Let $\kappa:[1, \infty) \rightarrow \mathbb{R}$ be a function. Throughout this section, by $\log (\cdot)$ we denote $\log _{e}(\cdot)$, and consider that $[\lambda]$ represents the integer part of a real number $\lambda$.

We also consider the following definitions:

1. The Hadamard derivative of fractional order $\lambda>0$ for $\kappa$ is

$$
D^{\lambda} \kappa(\theta)=\frac{1}{\Gamma(n-\lambda)}\left(\theta \frac{d}{d \theta}\right)^{n} \int_{1}^{\theta}\left(\log \frac{\theta}{u}\right)^{n-\lambda-1} \frac{\kappa(u)}{u} d u, n-1<\lambda<n ;
$$

2. The Hadamard fractional integral of order $\lambda>0$ for $\kappa$ is

$$
I^{\lambda} \mathcal{K}(\theta)=\frac{1}{\Gamma(\lambda)} \int_{1}^{\theta}\left(\log \frac{\theta}{u}\right)^{\lambda-1} \frac{\kappa(u)}{u} d u, \lambda>0,
$$

provided the integral exists.

In this section, we want to discuss the existence of a solution for the next fractional functional differential equation with initial values

$$
\left\{\begin{aligned}
D^{\lambda} \mathcal{K}(\theta) & =\varrho\left(\theta, \kappa_{\theta}\right), \text { for each } \theta \in[0, t], 0<\lambda<1 \\
\kappa(\theta) & =\zeta(\theta), \theta \in[1-z, 1]
\end{aligned}\right.
$$

with $\varrho:[1, t] \times C([-z, 0], \mathbb{R}) \rightarrow \mathbb{R}$ are given, $\zeta \in C([1-z, 1], \mathbb{R})$ is such that $\zeta(1)=0$ and for any $\vartheta, \kappa$ defined on $[1-y, t]$ the functions $\vartheta_{\theta}, \kappa_{\theta}$ are elements of $C([-z, 0], \mathbb{R})$ such that

$$
\kappa_{\theta}(\tau)=\kappa(\theta+\tau), \quad \vartheta_{\theta}(\tau)=\vartheta(\theta+\tau)
$$


for any $\theta \in[0, t]$. Let $\mathcal{M}=C([1-z, t], \mathbb{R})$ and $d: \mathcal{M} \times \mathcal{M} \rightarrow[0, \infty)$ defined as

$$
d(\kappa, \vartheta)=\sup _{\theta \in[1-z, t]}|\kappa(\theta)-\vartheta(\theta)|, \forall \kappa, \vartheta \in \mathcal{M},
$$

be the distance on the set $\mathcal{M}$ of real continuous functions .

Theorem 3. Let $a>0$ be such that $a \frac{(\log t)^{\lambda}}{\Gamma(\lambda+1)}<1$. Equation (25) has a solution on $[1-z, t]$ provided that for $\theta \in[1, t]$ and every $x, \kappa \in \mathcal{M}$

$$
|\varrho(\theta, \kappa)-\varrho(\theta, \chi)| \leq c \sup _{\theta \in[1, t]}|\kappa(\theta)-\vartheta(\theta)| .
$$

Proof. Consider a map $Z: \mathcal{M} \rightarrow \mathcal{M}$ defined by

$$
Z \kappa(\theta)=\left\{\begin{aligned}
\zeta(\theta), & \text { if } \theta \in[1-z, 1] \\
\frac{1}{\Gamma(\lambda)} \int_{1}^{\theta}\left(\log \frac{\theta}{u}\right)^{\lambda-1} \frac{\varrho\left(u, \kappa_{u}\right)}{u} d u, & \text { if } \theta \in[1, t] .
\end{aligned}\right.
$$

Thus, for $\theta \in[1, t]$ :

$$
\begin{aligned}
|Z \kappa(\theta)-Z \vartheta(\theta)| & \leq \frac{1}{\Gamma(\lambda)} \int_{1}^{\theta}\left(\log \frac{\theta}{u}\right)^{\lambda-1} \frac{\left|\varrho\left(u, \kappa_{u}\right)-\varrho\left(u, \vartheta_{u}\right)\right|}{u} d u \\
& \leq a \cdot \frac{1}{\Gamma(\lambda)} \int_{1}^{\theta}\left(\log \frac{\theta}{u}\right)^{\lambda-1} \sup _{\theta \in[1, t]}\left|\kappa_{u}-\vartheta_{u}\right| \frac{d u}{u} \\
& \leq a \cdot \frac{1}{\Gamma(\lambda)} \sup _{\theta \in[1-z, t]}\left|\kappa_{u}-\vartheta_{u}\right| \int_{1}^{\theta}\left(\log \frac{\theta}{u}\right)^{\lambda-1} \frac{d u}{u} \\
& =a \frac{(\log \theta)^{\lambda}}{\Gamma(\lambda+1)} \sup _{\theta \in[1-z, t]}\left|\kappa_{u}-\vartheta_{u}\right|,
\end{aligned}
$$

or, in other words

$$
d(T \kappa, T \vartheta) \leq a \frac{(\log \theta)^{\lambda}}{\Gamma(\lambda+1)} d(\kappa, \vartheta)
$$

Taking $\sigma_{1}=0, \sigma_{2}=1$ and $\psi(x)=a \frac{(\log \theta)^{\lambda} x}{\Gamma(\lambda+1)}$ for $x \geq 0$, the previous inequality becomes

$$
d(T \kappa, T \vartheta) \leq \psi\left(g_{T}^{s}(\kappa, \vartheta)\right)
$$

for any $s>0$. Thus, all the presumptions of Theorem 1 are satisfied and Equation (25) has a solution on $[1, t]$.

\section{Conclusions}

The obtained results unify several existing results in a single theorem. Herein, we have listed some of the consequences; however, it is clear that there are more consequences of our main results. As a result of the length of the paper, we have not included them here.

Author Contributions: All authors contributed equally and significantly in writing this article. All authors read and approved the final manuscript.

Funding: This research received no external funding.

Acknowledgments: The authors are grateful to the editor and to the reviewers for their valuable suggestions and comments.

Conflicts of Interest: The authors declare no conflict of interest. 


\section{References}

1. Liouville, J. Second mémoire sur le développement des fonctions ou parties de fonctions en séries dont divers termes sont assujettis á satisfaire a une m eme équation différentielle du second ordre contenant un paramétre variable. J. Math. Pure Appl. 1837, 2, 16-35.

2. Picard, E. Memoire sur la theorie des equations aux derivees partielles et la methode des approximations successives. J. Math. Pures Appl. 1890, 6, 145-210.

3. Banach, B. Sur les opérations dans les ensembles abstraits et leur application aux équations intégrales. Fundam. Math. 1992, 3, 133-181. [CrossRef]

4. Caccioppoli, R. Una teorema generale sull'esistenza di elementi uniti in una transformazione funzionale. Ren. Accad. Naz. Lincei 1930, 11, 794-799.

5. Iyiola, O.S.; Gaba, Y.U. On Metric Type Spaces and Fixed Point Theorems. Appl. Math. Sci. 2014, 79, 3905-3920. [CrossRef]

6. Gaba, Y.U. Fixed point theorems in G-metric spaces. J. Mat. Anal. Appl. 2017, 455, 528-537. [CrossRef]

7. Jaggi, D.S. Some unique fixed point theorems. Indian J. Pure Appl. Math. 1977, 8, 223-230.

8. Karapinar, E. Revisiting the Kannan Type Contractions via Interpolation. Adv. Theory Nonlinear Anal. Appl. 2018, 2, 85-87. [CrossRef]

9. Agarwal, R.P.; Karapinar, E. Interpolative Rus-Reich-Ciric Type Contractions Via Simulation Functions. An. Stiint. Univ. Ovidius Constanta Ser. Mat. 2019, in press.

10. Karapinar, E.; Alqahtani, O.; Aydi, H. On Interpolative Hardy-Rogers Type Contractions. Symmetry 2019, 11, 8. [CrossRef]

11. Karapinar, E.; Agarwal, R.P.; Aydi, H. Interpolative Reich-Rus-Ciric Type Contractions on Partial Metric Spaces. Mathematics 2018, 6, 256. [CrossRef]

12. Aydi, H.; Chen, C.M.; Karapinar, E. Interpolative Ciric-Reich-Rus Type Contractions via the Branciari Distance. Mathematics 2019, 7, 84. [CrossRef]

13. Aydi, H.; Karapinar, E.; Roldán López de Hierro, A.F. $\omega$ Interpolative Ciric-Reich-Rus Type Contractions. Mathematics 2019, 7, 57. [CrossRef]

14. Mitrović, Z.D.; Aydi, H.; Noorani, M.S.M.; Qawaqneh, H. The weight inequalities on Reich type theorem in b-metric spaces. J. Math. Comput. Sci. 2019, 19, 51-57.

15. Bianchini, R.M.; Grandolfi, M. Transformazioni di tipo contracttivo generalizzato in uno spazio metrico. Atti Acad. Naz. Lincei VII Ser. Rend. Cl. Sci. Fis. Mat. Nat. 1968, 45, 212-216.

16. Rus, I.A. Generalized Contractions and Applications; Cluj University Press: Cluj-Napocca, Romania, 2001. 